\title{
Multiscale Morphological Segmentations Based on Watershed, Flooding, and Eikonal PDE
}

\author{
Fernand Meyer ${ }^{1}$ and Petros Maragos ${ }^{2}$
}

1 Centre de Morphologie Mathématique, Ecole des Mines de Paris, 35, Rue Saint Honoré, 77305 Fontainebleau, France. Email: meyer@cmm.ensmp.fr

2 National Technical University of Athens, Dept. of Electrical \& Computer Engineering, Zografou 15773, Athens, Greece. Email: maragos@cs.ntua.gr

\begin{abstract}
The classical morphological segmentation paradigm is based on the watershed transform, constructed by flooding the gradient image seen as a topographic surface. For flooding a topographic surface, a topographic distance is defined from which a minimum distance algorithm is derived for the watershed. In a continuous formulation, this is modeled via the eikonal PDE, which can be solved using curve evolution algorithms. Various ultrametric distances between the catchment basins may then be associated to the flooding itself. To each ultrametric distance is associated a multiscale segmentation; each scale being the closed balls of the ultrametric distance.
\end{abstract}

\section{Introduction}

Segmentation is one of the most challenging tasks in image processing, as it requires to some extent a semantic understanding of the image. The morphological segmentation paradigm, based on the watershed transform and markers, has been extremely successful, both for interactive as for automatic segmentation. Its principle is simple: a) a gradient image of the scene is constructed; b) for each object of interest, an inside particle is detected, either in an automatic manner or in an interactive manner; c) construction of the watershed associated to the markers. Its avantage is the robustness: the result is independent of the shape or the placement of the markers in the zones of interest. The result is obtained by a global minimization implying both the topography of the surface and the complete set of markers.

This paradigm has met its limits with the emergence of new segmentation tasks in the area of communications and multimedia industry. The development of games, teleworking, teleshopping, television on demand, videoconferences etc. has multiplied situations where images and sequences have not only to be transmitted but also manipulated, selected, assembled in new ways. This evolution is most challenging for segmentation techniques: one has to segment complex sequences of color images in real time, be automatic but also able to deal with user interaction.

Object oriented coding represents an even greater challenge for segmentation techniques. Such encoders segment the scene into homogeneous zones for which 
contours, motion and texture have to be transmitted. Depending upon the targeted bitstream and the complexity of the scene, a variable number of regions has to be transmitted. Hence an automatic segmentation with a variable number of regions is required for sequences for which the content or even content type is not known a priori. Hence, there is no possibility to devise a strategy for finding markers, and as a consequence the traditional morphological segmentation based on watershed and markers fails.

This situation has triggered the development of new techniques of multiscale segmentation, where no markers are required. In such cases it is of interest to construct a sequence of nested partitions going from coarse to fine; each boundary of a coarse segmentation also being a boundary of all finer segmentations. We will call such a series of nested partitions a multiscale cube (we do not call it pyramid, as the resolution of the images is not reduced when going from fine to coarse). Such a multiscale cube may be used in various ways:

- chose in the cube a slice with the appropriate number of regions

- compose a segmentation by extracting regions from different slices of the cube. This may be done in an interactive way. It may also result by minimizing some global criterion (for instance, if a texture model is adopted for each region, it is possible to measure the distance between the model and the original image in each region. It is then possible to minimize a weighted sum of the length of the contours and of the global distortion of the image).

- use the pyramid for defining new dissimilarity measures between the adjacent catchment basins, which may be used for segmenting with markers and yield better results as the traditional segmentation with markers, using the altitude of the gradient.

In absence of any knowledge of the image content, it is important to find good psychovisual criteria for constructing the cube.

In this paper, we first discuss the monoscale watershed segmentation by flooding both from a discrete formulation of the shortest topographic distance as well as from a continuous viewpoint of the eikonal PDE and curve evolution. Further, for multiscale segmentation, we use ultrametric distances to generalize the flooding and improve the segmentation.

\section{The classical morphological segmentation paradigm}

\subsection{Flooding a topographic surface}

The classical morphological tool for segmentation is the watershed transform. For segmenting an image $f$, first its edges are enhanced by computing its gradient magnitude $\|\nabla f\|$. This is approximated by the discrete morphological gradient $\delta(f)-\varepsilon(f)$, where $\delta(f)=f \oplus B$ is the flat dilation of $f$ by a small disk $B$ and $\varepsilon(f)=f \ominus B$ is the flat erosion of $f$ by $B$. After the edge enhancement, the segmentation process starts with creating flooding waves that emanate from a set of markers (feature points inside desired regions) and flood the topographic 
surface $\|\nabla f\|$. The points where these flooding waves meet each other form the segmentation boundaries. The simplest markers are the regional minima of the gradient image. Very often, the minima are extremely numerous, leading to an oversegmentation. For this reason, in many practical cases, the watershed will take as sources of the flooding a smaller set of markers, which have been identified by a preliminary analysis step as inside germs of the desired segmentation.

\subsection{Modifying a topographic surface: Swamping}

In the case where the sources for the flooding are not all minima of the topographic surface, two solutions are possible. Either use the markers as sources. In this case, catchement basins without sources are flooded from already flooded neighbouring region. Such a flooding algorithm, using hierarchical queues has been described in [1].

The second solution consists in modifying the topographic surface as slightly as possible, in such a way that the markers become its only regional minima. This operation is called swamping. If $m_{1}, m_{2}, \ldots m_{k}$ are the binary markers we construct a marker function $g$ defined as follows : $g=$ White outside the markers and $g=$ Black inside the markers. On the other hand, the topographic surface $f$ is modified by assigning the value Black to all regional minima. We then perform a closing by reconstruction of $f$ from the marker function $g$. This can be accomplished by an iterative algorithm which at each iteration forms a conditional erosion, i.e., a supremum $(\vee)$ of the erosion of the previous iterate and the original function:

$$
\begin{aligned}
& g_{0}=g \vee f \\
& g_{k}=\varepsilon\left(g_{k-1}\right) \vee f \quad, \quad k=1,2,3, \ldots
\end{aligned}
$$

In the limit as $k \rightarrow \infty$ we obtain the function $g_{\infty}$ which is the result of the closing by reconstruction. This new function is as similar as possible to the function $f$, except that its only regional minima are the family $\left\{m_{i}\right\}$. Hence, its catchment basins will give the desired segmentation.

\section{Watershed Segmentation: Discrete and Continuous}

\subsection{Discrete Watershed and Topographic Distance}

We consider first images in a digital framework. Images are represented on regular graphs where the nodes represent the pixels and the edges the neighborhood relations. A connected component of uniform grey tone is called plateau. A plateau without lower (resp. higher) neighbors is a regional minimum (resp. maximum).

Let us now consider a drop of water falling on a topographic surface $f$ for which the regional minima are the only plateaus. If it falls outside a plateau, it will glide along a path of steepest descent. If the altitude of a pixel $x$ is $f(x)$, the altitude of its lowest neighbor defines the erosion $\varepsilon(f)(x)$ of size 1 



Fig. 1. (a) Initial topographic surface. (b) Creation of the marker. (c) Result of the swamping.

at pixel $x$. Hence the altitude of the steepest descending slope at pixel $x$ is slope $(x)=f(x)-\varepsilon(f)(x)$. If $x$ and $y$ are two neighboring pixels, we will define the topographic variation topvar $(x, y)$ between $x$ and $y$ as slope $(x)$ if $f(x)>f(y)$ and as $\frac{\operatorname{slope}(x)+\operatorname{slope}(y)}{2}$ if $f(x)=f(y)$.

If $\pi$ is a path $\left(x=p_{1}, p_{2}, \ldots, y=p_{n}\right)$ between two pixels $x$ and $y$, we define the topographical variation along the path $\pi$ as the sum $\sum_{i=1, n-1} \operatorname{topvar}\left(p_{i}, p_{i+1}\right)$ of the elementary topographical variations along the path $\pi$. The topographical distance between two pixels $x$ and $y$ is defined as the minimal topographical variation along all paths between $x$ and $y$. By construction, the trajectory of a drop of water falling on the surface is a geodesic line of the topographic distance. A pixel $p$ belongs to the upstream of a pixel $q$ if and only if the topographic distance between both pixels is equal to $|f(p)-f(q)|$. Let us now transform the topographic surface by putting all regional minima at altitude 0 .

Definition 1. We call catchment basin $\mathrm{CB}\left(m_{i}\right)$ of a regional minimum $m_{i}$ the set of pixels which are closer to $m_{i}$ than to any other regional minimum for the topographical distance

A more general description of the topographic distance, also valid for images with plateaus may be found in [7. Within each catchment basin, the set of pixels closer to the minimum than a given topographic distance $h$ are all pixels of this basin with an altitude below $h$. In this framework the construction of the catchment basins becomes a shortest path problem, i.e., finding the path between a marker and an image point that corresponds to the minimum weighted distance. Computing this minimum weighted distance at all image points from any marker is also equivalent to finding the gray-weighted distance transform (GWDT) of the image. There are several types of discrete algorithms to compute the GWDT which include iterated (sequential or parallel) min-sum differences [13] and hierarchical queues [7]. Instead of elaborating more on discrete GWDT algorithms, we prefer now to proceed to our next formulation of watershed that will be based on a continuous (PDE-based) model. Afterwards, the discrete GWDT 
will be re-interpreted as one possible discrete approximation to the solution of the continuous problem.

\subsection{Continuous Watershed and Eikonal PDE}

The watershed transforms an image $f(x, y)$ to the crest lines separating adjacent catchment basins that surround regional minima or other 'marker' sets of feature points. In a continuous formulation, the topographic distance of $f$ along a path becomes the line integral of $\|\nabla f\|$ along this path. Viewing the domain of $f$ as a $2 \mathrm{D}$ optical medium with a refractive index field $\eta(x, y)=\|\nabla f\|$, makes the continuous topographic distance function equivalent to the optical path length which is proportional to the time required for light to travel this path. This leads to the eikonal PDE

$$
\|\nabla U(x, y)\|=\eta(x, y), \quad \eta(x, y)=\|\nabla f(x, y)\|
$$

whose solution for any field $\eta(x, y)$ is a weighted distance function [112]. In the continuous domain and assuming that the image is smooth and has isolated critical points, the continuous watershed is equivalent to finding a skeleton by influence zones with respect to a weighted distance function that uses points in the regional minima of the image as sources and $\eta=\|\nabla f\|$ as the field of indices [97]. If other markers different than the minima are to be used as sources, then the homotopy of the function must be modified via morphological reconstruction to impose these markers as the only minima.

Modeling the watershed via the eikonal has the advantage of a more isotropic flooding but also poses some challenges for its implementation. This problem can be approached by viewing the solution of the eikonal PDE as a gray-weighted distance transform (GWDT) whose values at each pixel give the minimum distance from the light sources weighted by the gray values of the refractive index field. Next we outline two ways of solving the eikonal PDE as applied to segmentation.

\subsection{GWDT based on Chamfer Metrics}

Let $\eta[i, j]$ be a sampled nonnegative gray-level image and let us view it as a discrete refractive index field. Also let $S$ be a set of reference points or the 'sources' of some wave or the location of the wavefront at time $t=0$. As discussed earlier, the GWDT finds at each pixel $p=[i, j]$ the smallest sum of values of $\eta$ over all possible paths connecting $p$ to the sources $S$.

This discrete GWDT can be computed by running a 2D min-sum difference equation like the one implementing the chamfer distance transform of binary images but with spatially-varying coefficients proportional to the gray image values 13:

$$
\begin{aligned}
& U_{k}[i, j]=\min \left\{U_{k}[i-1, j]+a \eta[i, j], U_{k}[i, j-1]+a \eta[i, j],\right. \\
& \left.\quad U_{k}[i-1, j-1]+b \eta[i, j], U_{k}[i-1, j+1]+b \eta[i, j], U_{k-1}[i, j]\right\}
\end{aligned}
$$


where $U_{0}$ is the $0 / \infty$ indicator function of the source set $S$. Starting from $U_{0}$, a sequence of functions $U_{k}$ is iteratively computed by running (3) over the image domain in a forward scan for even $k$, whereas for odd $k$ an equation as in (3) but with a reflected coefficient mask is run in a backward scan. In the limit $k \rightarrow \infty$ the final GWDT $U_{\infty}$ is obtained. In practice, this limit is reached after a finite number of passes. The above implementation can also be viewed as a procedure of finding paths of minimal 'cost' among nodes of a weighted graph or as discrete dynamic programming. As such it is actually known as Dijkstra's algorithm. There are also other faster implementations using queues 136]. The above GWDT based on discrete chamfer metrics is shown in [13] and [4] to be a discrete approximate solution of the eikonal PDE $\|\nabla U\|=\eta$.

The constants $a$ and $b$ are the distance steps by which the planar chamfer distances are propagated within a $3 \times 3$ neighborhood. To improve the GWDT approximation to the eikonal's solution, one can optimize $(a, b)$ to minimize the error between the chamfer and Euclidean distances and/or use larger neighborhoods (at the cost of a slower implementation). However, using a neighborhood larger than $5 \times 5$ may give erroneous results since the large masks can bridge over a thin line that separates two segmentation regions. Overall, this chamfer metric approach to GWDT is fast and easy to implement, but due to the required small neighborhoods is not isotropic and cannot achieve high accuracy.

\subsection{GWDT based on Curve Evolution}

In the standard digital watershed algorithm 814, the flooding at each level is achieved by a planar distance propagation that uses the chess-board metric. This kind of distance propagation is non-isotropic and could give wrong results, particularly for images with large plateaus, as we found experimentally. Eikonal segmentation using GWDTs based on chamfer metrics improves this situation a little but not entirely. In contrast, for images with large plateaus/regions, segmentation via the eikonal PDE and curve evolution GWDT gives results close to ideal.

In the PDE-based watershed approach [5], at time $t=0$ the boundary of each source is modeled as a curve $\gamma(0)$ which is then propagated with normal speed $c(x, y)=c_{0} / \eta(x, y)=c_{0} /\|\nabla f(x, y)\|$, where $c_{0}$ is the largest constant speed (e.g., the speed of light in vacuum). The propagating curve $\gamma(t)$ is embedded as the zero-level curve of a function $F(x, y, t)$, where $F(x, y, 0)=F_{0}(x, y)$ is the signed (positive in the curve interior) distance from $\gamma(0)$. The function $F$ evolves according to the PDE

$$
\frac{\partial F}{\partial t}=c(x, y)\|\nabla F\|
$$

As analyzed in 1012, this PDE implies that all the level curves of $F$ propagate with a position-dependent normal speed $c(x, y)>0$. This is a time-dependent formulation of the eikonal PDE and can be solved via the entropy condition satisfying numerical algorithm of [10]. The value of the resulting GWDT at any pixel $(x, y)$ of the image is the time it takes for the evolving curve to reach this 
pixel, i.e. the smallest $t$ such that $F(x, y, t) \geq 0$. The wateshed is then found along the lines where wavefronts emanating from different markers collide and extinguish themselves.

To reduce the computational complexity of solving general eikonal PDE problems via curve evolution a 'fast marching' algorithm was developed in 123 , that tracks only a narrow band of pixels at the boundary of the propagating wavefront. For the eikonal PDE segmentation problem, a queue-based algorithm has been developed in $[5$ that combines features from the fast marching method to computing GWDTs and can deal with the case of multiple sources where triple points develop at the collision of several wavefronts.

As Fig. 2 shows, compared on a test image that is difficult (because expanding wavefronts meet watershed lines at many angles ranging from being perpendicular to almost parallel), the continuous segmentation approach based on the eikonal PDE and curve evolution outperforms the discrete segmentation results (using either the digital watershed flooding algorithm or chamfer metric GWDTs). However, some real images may not contain many plateaus or only large regions, in which cases the digital watershed flooding algorithm may give comparable results than the eikonal PDE approach.

\section{Ultrametric distances associated to flooding}

\subsection{Ultrametric distance and multiscale partitions}

The first part of the paper has described the tools for producing the finest partition, from which a multiscale representation may be derived. Let $P_{0}=$ $\left(P_{01}, P_{02}, \ldots P_{0 n}\right)$ be the list of regions forming the finest partition. We are interested in constructing a series of nested partitions $P_{k}=\left(P_{k 1}, P_{k 2}, \ldots P_{k n}\right)$, where each region $P_{k j}$ is the union of a number of regions of finer partitions $P_{l}$, for $l<k$.

It is classical to associate to the series of nested partitions $\left(P_{k}\right)$ an ultrametric distance :

$d\left(P_{0 i}, P_{0 j}\right)=\min \left(l \mid \exists P_{l h} \in P_{l}\right.$ for which $P_{0 i} \subset P_{l h}$ and $\left.P_{0 j} \subset P_{l h}\right)$. In other words, the ultrametric distance is the smallest index of a partition $P_{h}$, of which one of the sets $P_{l h}$ contains both regions $P_{0 i}$ and $P_{0 j}$.

It is an ultrametric distance as it verifies the following axioms :

* reflexivity $: d\left(P_{0 i}, P_{0 i}\right)=0$

* symmetry: $d\left(P_{0 i}, P_{0 j}\right)=d\left(P_{0 j}, P_{0 i}\right)$

* ultrametric inequality : for all $i, j, k$ : $d\left(P_{0 i}, P_{0 j}\right) \leq \max \left\{d\left(P_{0 i}, P_{0 k}\right), d\left(P_{0 k}, P_{0 j}\right)\right\}$

The first two axioms are obviously verified. The last one may be interpreted as follows : the smallest index $l$ of a region $P_{l h}$ containing both regions $P_{0 i}$ and $P_{0 j}$ is necessarily smaller or equal than the smallest index $u$ of a region $P_{u v}$ containing all three regions $P_{0 i}, P_{0 j}$ and $P_{0 k}$

An ultrametric distance is a distance, as the ultrametric inequality is stronger than the triangular inequality. A closed ball for the ultrametric distance with 


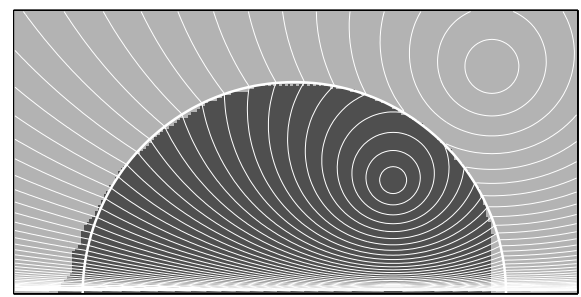

(a)

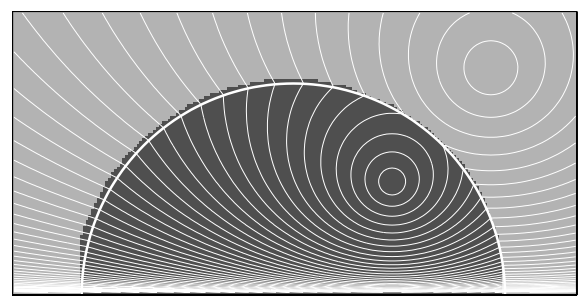

(c)

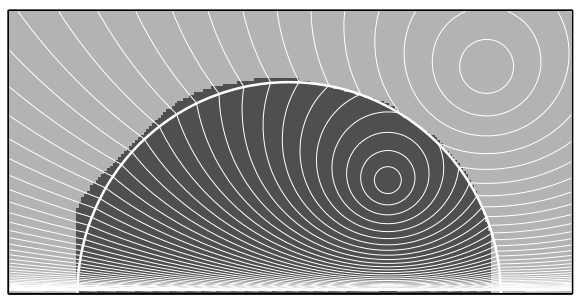

(b)

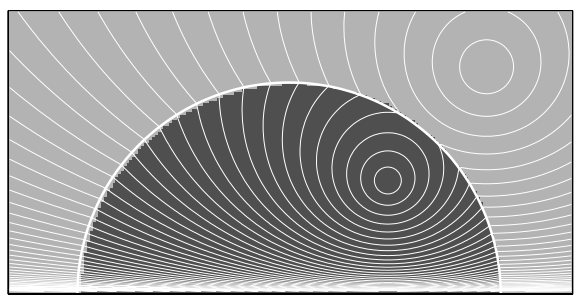

(d)

Fig. 2. Performance of various segmentation algorithms on a TEST image $(250 \times 400$ pixels). This image is the minimum of two potential functions. Its contour plot (thin bright curves) is superimposed on all segmentation results. Markers are the two source points of the potential functions. Segmentation results based on: (a) Digital watershed flooding algorithm. (b) GDWT based on optimal $3 \times 3$ chamfer metric. (c) GDWT based on optimal $5 \times 5$ chamfer metric. (d) GDWT based on curve evolution. (The thick bright curve shows the correct segmentation.)

centre $P_{0 k}$ and radius $n$ is the set of all regions $P_{0 i}$ for which $d\left(P_{0 i}, P_{0 j}\right) \leq n$. The balls associated to an ultrametric distance have two unique features, which will be useful in segmentation. The radius of a ball is equal to its diameter, i.e. to the largest distance between two elements in the ball. Each element of a ball is the centre of this ball. It is easy to check that the union of all closed balls of radius $n$ precisely constitute the partition $P_{n}$.

Inversely we will associate a series of nested partitions to each ultrametric distance, by taking for partition of rank $n$, the set of closed balls of radius $n$. We will now define several ultrametric distances, naturally associated to the flooding of a topographic surface. Each of them will yield a different partition cube. 


\subsection{Flooding Tree}

A finer analysis of the flooding will show the apparition of a tree in which the nodes are the catchment basins and the edges represent relations between neighboring nodes. Let us observe the creation and successive fusions of lakes during the flooding. The level of the flood is uniform over the topographic surface and increases with constant speed : new lakes appear as the flood reaches the various regional minima. At the time of apparition, each lake is isolated. As the level increases and reaches the lowest passpoint separating the corresponding CB from a neighboring CB, two lakes will merge. Two types of passpoints are to be distinguished. When the level of the flood reaches the first type, two lakes previously completely disconnected merge ; we will call these passpoints first meeting passes. When the flood reaches the second type, two branches of a unique lake meet and form a closed loop around an island. Representing each first meeting pass as an edge of a graph and the adjacent catchment basins as the nodes linked by this edge will create a graph. It is easy to see that this graph is a tree, spanning all nodes. It is in fact the minimum spanning tree (MST) of the neighborhood graph obtained by linking all neighboring catchment basins by an edge weighted by the altitude of the passpoint between them.

\subsection{Flooding via Ultrametric Distances}

Each edge of the spanning tree represents a passpoint where two disconnected lakes meet. We will assign to this edge a weight derived by measuring some geometric features on each of the adjacent lakes. We consider four different measures. The simplest is the altitude of the passpoint itself. The others are measured on each lake separately : they are respectively the depth, the area and the volume of the lakes For each of these four types a weight is derived as follows. Let us consider for instance the volume : the volumes of both lakes are compared and the smallest value is chosen as volumic weight of the edge. Depth and area measures are treated similarly leading respectively to weight distributions called dynamics for the depth and surfacic weight distributions. If the height is chosen, we get the usual weight distribution of the watershed.

We will now define an ultrametric distance associated to each weight distribution on the MST : the distance $d(x, y)$ is defined as the highest weight encountered on the unique path going from $x$ to $y$ along the spanning tree. This relation obviously is reflexive and symmetrical. The ultrametric inequality also is verified : for all $x, y, z, d(x, y) \leq \max \{d(x, z), d(z, y)\}$; since the highest weight on the unique path going from $x$ to $y$ along the spanning tree is smaller or equal to the highest way on the unique path which goes first from $x$ to $z$ and then from $z$ to $y$ along the spanning tree.

The closed balls of the ultrametric distance precisely correspond to the segmentation tree induced by the minimum spanning tree. The balls of radius 0 are the individual nodes, corresponding to the catchment basins. Each ball of radius $n$ is the union of all nodes belonging to one of the subtrees of the MST obtained by cutting all edges with a valuation higher than $n$. A closed ball of 
radius $R$ and centre $C$ is the set of nodes which belong to the same subtree of the MST, obtained by cutting the edges at altitude higher than or equal to $R$ and containing $C$. Obviously replacing the centre $C$ by any other node of the subtree yields the same subtree.

Cutting the $(k-1)$ highest edges of the minimum spanning tree creates a forest of $k$ trees. This is the forest of $k$ trees of minimal weight contained in the neighborhood graph. Depending on the criterion on which the ultrametric distance is based, the nested segmentations will be more or less useful. The ultrametric distance based on altitude is the less useful. The segmentation based on depth are useful for ranking the particles according to their contrast. The area ultrametric distance will focus on the size of the particles. The volumic ultrametric distance has particularly good psychovisual properties [15]: the resulting segmentation trees offer a good balance between size and contrast. as illustrated in the following figures. The topographical surface to be flooded is a color gradient of the initial image (maximum of the morphological gradients computed in each of the R, G and B color channels). The volumic ultrametric distance has been used, and 3 levels of fusions have been represented, corresponding respectively to 15,35 and 60 regions.

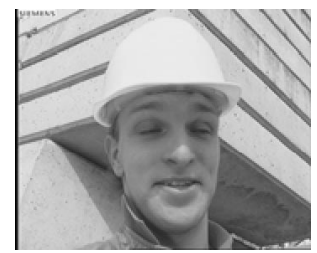

Initial

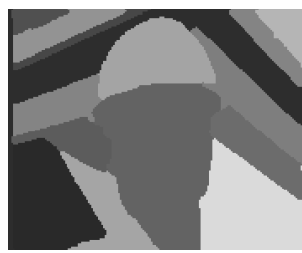

15 regions

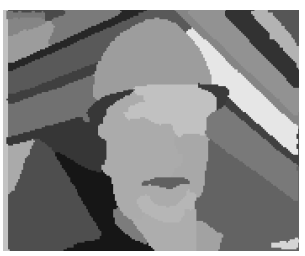

35 regions

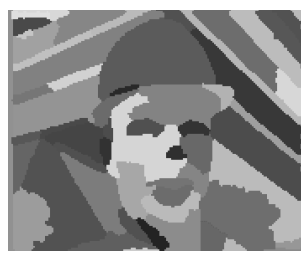

60 regions

Fig. 3. Multiscale segmentation example.

\section{Applications}

\subsection{Interactive segmentation with nested segmentations}

A toolbox for interactive editing is currently constructed at the CMM [16, based on nested segmentations. A mouse position is defined by its $\mathrm{x}-\mathrm{y}$ coordinates and its depth in the segmentation tree. If the mouse is active, the whole tile containing the cursor is activated. Moving the mouse in the $\mathrm{x}-\mathrm{y}$ plane permits to select or deselect regions at the current level of segmentation. Going up will produce a coarser region, going down a smaller region. This technique permits to "paint" the segmentation with a kind of brush, whose shape adapts itself to the contours and whose size may be interactively changed by the user. 


\section{The watershed from markers}

In many situations one has a seed for the objects to segment. It may be the segmentation produced in the preceding frame when one has to track an object in a sequence. It may also be some markers produced by hand, in interactive segmentation scenarios. As a result, some nodes of the minimum spanning tree may be identified as markers. The resulting segmentation associated to these markers will then still be a minimum spanning forest, but constrained in that each tree is rooted in a marker. The algorithm for constructing the minimum spanning forest is closely related to the classical algorithms for constructing the MST itself (see ref[17]. for more details). Each marker gets a different label and constitutes the initial part of a tree. The edges are ranked and processed in increasing order. The smallest unprocessed edge linking one of the tree $T$ to an outside node is considered ; if this node does not already belong to another tree, it is assigned to the tree $T$. If it belongs to another tree, the edge is discarded and the next edge is processed.

Segmenting with markers constitutes the classical morphological method for segmentation. For optimal results, it is important to correctly chose the underlying ultrametric distance. We have presented 3 new distances giving often better results than the classically used flooding distance (where the weights are the altitude of the passpoints) This is illustrated by the following figures, where the same set of markers has been used alternatively with the flooding distance and with the volumic distance. The superiority of the volumic distance clearly appears here : it correctly detects the face, whereas the flooding distance follows the boundary of a shadow and cuts the face in two.

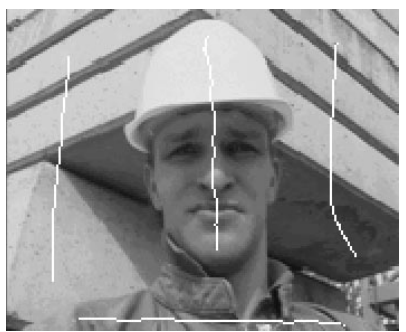

markers

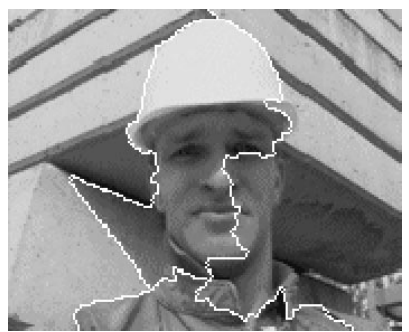

flooding dist.

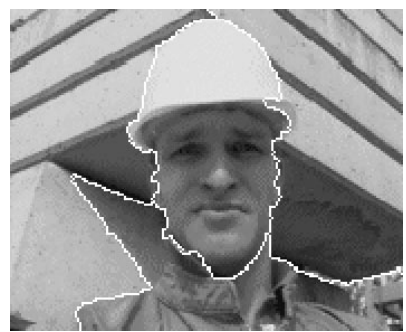

volumic dist.

Fig. 4. Segmentations with different ultrametric floodings.

\section{Conclusion}

A multiscale segmentation scheme has been presented, embedded in the flooding mechanism of the watershed itself. It opens many new possibilities for segmentation, either in supervised or unsupervised mode. 
Acknowledgements: P. Maragos' work in this paper was partially supported by the European TMR/Networks Project ERBFMRXCT970160.

\section{References}

1. S. Beucher and F. Meyer. The morphological approach to segmentation: the watershed transformation. In E. Dougherty, editor, Mathematical morphology in image processing, chapter 12, pages 433-481. Marcel Dekker, 1993.

2. R. Kimmel, N. Kiryati, and A. M. Bruckstein, "Sub-Pixel Distance Maps and Weighted Distance Transforms", J. Math. Imaging \& Vision, 6:223-233, 1996.

3. R. Malladi, J. A. Sethian, and B. C. Vemuri, "A Fast Level Set Based Algorithm for Topology-Independent Shape Modeling", J. Math. Imaging and Vision, 6, pp. 269289, 1996.

4. P. Maragos, "Differential Morphology and Image Processing" IEEE Trans. Image Processing, vol. 78, pp. 922-937, June 1996.

5. P. Maragos and M. A. Butt, "Advances in Differential Morphology: Image Segmentation via Eikonal PDE \& Curve Evolution and Reconstruction via Constrained Dilation Flow", in Mathematical Morphology and Its Applications to Image and Signal Processing, H. Heijmans and J. Roerdink, Eds., Kluwer Acad. Publ., 1998, pp. $167-174$.

6. F. Meyer, "Integrals and Gradients of Images", Proc. SPIE vol. 1769: Image Algebra and Morphological Image Processing III, pp.200-211, 1992.

7. F. Meyer, "Topographic Distance and Watershed Lines", Signal Processing, 38, pp. 113-125, 1994.

8. F. Meyer and S. Beucher, "Morphological Segmentation", J. Visual Commun. Image Representation, 1(1):21-45, 1990.

9. L. Najman and M. Schmitt, "Watershed of a Continuous Function", Signal Processing, vol. 38, pp. 99-112, July 1994.

10. S. Osher and J. Sethian, "Fronts Propagating with Curvature-Dependent Speed: Algorithms Based on Hamilton-Jacobi Formulations", J. Comput. Physics, 79, pp. 12-49, 1988.

11. E. Rouy and A. Tourin, "A Viscocity Solutions Approach to Shape from Shading", SIAM J. Numer. Anal., vol. 29 (3), pp. 867-884, June 1992.

12. J. A. Sethian, Level Set Methods, Cambridge Univ. Press, 1996.

13. P. Verbeek and B. Verwer, "Shading from shape, the eikonal equation solved by grey-weighted distance transform", Pattern Recogn. Lett., 11:618-690, 1990.

14. L. Vincent and P. Soille, "Watershed In Digital Spaces: An Efficient Algorithm Based On Immersion Simulations", IEEE Trans. Pattern Anal. Mach. Intellig., vol. 13, pp. 583-598, June 1991.

15. C. Vachier. Extraction de Caractéristiques, Segmentation d'Image et Morphologie Mathématique. PhD thesis, E.N.S. des Mines de Paris, 1995.

16. F. Zanoguera, B. Marcotegui and F. Meyer, "An interactive colour image segmentation system", Wiamis'99: Workshop on Image Analysis for Multimedia Interactive Services, pp.137-141. Heinrich-Hertz Institut Berlin, 1999.

17. F. Meyer. Minimal spanning forests for morphological segmentation. ISMM94: Mathematical Morphology and its applications to Signal Processing, pages 77-84, 1994. 